\title{
1. Introduction: academic commitment and leadership as a model for the 21 st-century university
}

Universities are undergoing dramatic changes - from exponential expansion of student enrolments and a proliferation of university foundings, to new technological means for teaching and research, to massive reforms of their governance and management - and such changes not only threaten the traditional view of the university as the prime knowledge organization but also reconfigure its relations with society. For decades, universities have been battling their image as the "ivory tower of academia", referring to elitist and insular organizations that are disconnected from society's challenges and needs. However, the university has a long history of engagement with society, which includes providing both the education and the forum to transform students into productive citizens as well as stimulating discoveries that have broadened human horizons.

The historiography of the university is, indeed, understood through the evolutionary process of the university's mission. The purpose of the university is informed by internal, institutional ambitions and by the external notion of the good life, and thus its transformation should be appreciated while keeping this duality of impetus in mind. Newer fundamental goals did not supplant the university's previous mission; rather, they emerged as new centres of purpose while integrating prior concerns as auxiliary to the mission. The university's mission metamorphosis began, at the outset, with the initial goal of teaching that defined the medieval university. The second mission, that of research, was introduced in the early 19th century to form the Humboldtian model of the university. Subsequently, a third mission of commercialization was introduced in the mid-20th century to create the entrepreneurial university.

During each of these epochal models of the university - a teaching organization, a knowledge-generating organization, and then also a productive organization - university-society relations were redefined. 
Throughout the reformulations of the university's mission, and through generations of struggles to secure the autonomy of these institutions from political and economic pressures (while still reactive to these external stimuli as they inform the societal values $d u$ jour), universities have continuously been engaged with society, albeit it in different forms. Still, the spirit of 21 st-century global society demands the university take on a new central aim: it requires that universities take social responsibility, adopt a leadership position, and have social impact.

These days, the language to describe a university's social role is changing worldwide, expressing a further responsibility of universities to expand their impact on society either economically, socially (broadly and thus ambiguously defined), or ecologically. Numerous indexes used to assess and compare universities have recently added dimensions that express the wish for universities to expand their impact on society. For example, several transnational initiatives are now organizations valorizing the social impact action of universities, thus setting academic commitment and leadership (ACL) to social causes as a normative and operational expectation. Two such initiatives were launched in 2019, spurring this normative change among both academicians and universities, on both the individual and organizational levels, while also encouraging the public to seek social yield from academic research. One is the European Commission's new Horizon Impact Award: research projects that received FP-7 or Horizon 2020 funding are eligible for additional recognition for the "use of their results to provide value for society". The second is the Times Higher Education's new ranking of universities by their performance towards the social goals encoded as the United Nations' Sustainable Development Goals (SDGs). Our claims in this book are aligned with the spirit of these initiatives.

The demands upon universities to rethink their social role are captured in several iconic documents at every turning point. For instance, Vannevar Bush's 1945 Science - The Endless Frontier has been made sacred in expressing the economic commitments of universities towards the development of society's security and prosperity and thus the traditional "third mission" and "entrepreneurial university". Recently, such documents are emerging, delivering 21 st-century expectations from the university. One such document is "The Communique of the 2009 World Conference on Higher Education", which was convened in Paris by UNESCO. This 2009 communique articulates these new challenges laid before universities; it unequivocally speaks of the social responsibility of 
higher education - that is, institutions and individuals alike. Decreeing this position, the document states the following:

Faced with the complexity of current and future global challenges, higher education has the social responsibility to advance our understanding of multifaceted issues, which involve social, economic, scientific and cultural dimensions and our ability to respond to them. It should lead society in generating global knowledge to address global challenges, inter alia food security, climate change, water management, intercultural dialogue, renewable energy and public health. (Item 2)

With these words, the communique not only calls for academic responsibility but also expresses a sense of urgency due to the mounting challenges facing societies worldwide at the start of the 21 st century. How are universities and academicians to respond to such expectations? How are they to balance societal expectations for scientific input with maintaining the core principles that secured the university's resilience over a thousand years? Therefore, what is the appropriate social role of universities under the new social conditions of the current era? The premise of our book is to suggest a path for securing the university's role as a socially engaged institution, expressing its social commitment, and taking its role as a leader towards social change. We therefore propose the model we call ACL - academic commitment and leadership - as the format for redefining the social role of universities and transforming university-society relations in a manner that befits our times.

\section{DEFINING ACADEMIC COMMITMENT AND LEADERSHIP (ACL)}

In this book, we introduce, define, and analyse ACL. We argue that ACL, as implied by its title, reorients discussions of academia's social role and the impact of universities on the public good towards commitment and leadership. Therefore, the definition of ACL is a model for universitysociety relations that demands the socialization of academicians to pledge themselves to the advancement and protection of the public good, as well as stressing the importance of organizational reforms to facilitate this reorientation. ACL is constituted around three main themes:

1. Advancing the public good and social issues, allowing for a broad spectrum of targets and courses; 
2. Fostering academic leadership of social impact and change, driven by both academicians and scientists as mainly a "bottom-up" process of responsibility and action; and

3. Institutionalizing structures and practices that encourage ACL and socialize new entrants into such a culture, yet also pertaining to the three existing missions of academia.

Together, the three defining elements weave a description of the goal (the public good), the actors (academicians), and the form (institutionalization of new norms with regards to the new mission). Importantly, this definition clarifies two distinct characteristics of ACL, compared with existing initiatives taken by universities and academicians to impact society. First, ACL's commitment to, and leadership of, social causes is distinct from the focus on economic or technological development, which stands firmly as the core of academia's third mission. Rather, as we define it, the proposed model of ACL orients academic commitment towards the advancement of the public good and social agenda and towards fostering leadership of social change as a distinct and integral goal of academic education (see Chapters 2 and 3). Second, ACL is driven by academicians and scientists; hence, it is mostly a bottom-up model for responsibility for the public good. Academia's leaders and managers should facilitate ACL by setting guidelines that encourage ACL themes and establishing programmes that enable ACL initiatives to flourish; nevertheless, ACL demands the socialization of academicians to pledge themselves to the advancement and protection of the public good. In this sense, although the engagement of universities with the societies in which they were and are embedded has old roots and many varieties, these university-society relations are predicated on the notion of the public (see Holmwood, 2011).

Seeing that the outlined ACL model relies on the initiatives of individual academicians, whereas universities are there to offer support and empower such activities, an important question is illuminated in stage lights: what is a socially responsible academic leader? We think that the definition of this hybrid identity is best explained by two concepts that originate from social network theories: "leveraging" and "brokerage".

Leverage is a positional advantage that provides power and ability to act effectively and influence people (Quinn, 1999; Quinn et al., 1996). In the context of ACL, leveraging refers to the ability of an academician to use the advantage associated with her or his reputable social position to create advantage in gaining certain socially oriented goals that she or he believes in and wishes to promote. Through leveraging, "ACL-ers" allow 
themselves to be associated with a public cause and to then steer attention and actions towards the advancement of this public cause. To create such an effect, the ACL-driven academician uses his or her reputation and renown as a scientist or academic official.

Brokerage, on the other hand, is a structural position that is able to bridge or bind two social domains that are otherwise disconnected (Burt, 2004, 2005). Being in such a brokerage position can benefit both the person in that position and the domains he or she connects: effective brokerage may bring great returns to the people occupying a brokerage position, as well as in terms of having them achieve their goals, and it may result in high innovation in the connected domains because of the transfer or crossing of ideas and practices between these otherwise distinct and separate social or organizational contexts (Burt, 2007). Therefore, for ACL and in general, brokerage position does not uniquely connect among individuals, but it can also connect between fields of action, social groups, organizational fields, and alike social entities. Through brokerage, an ACL-motivated academician harnesses resources from unconnected domains and enlarges the pool of possibilities by creating synergies and multiplication effects through the link he or she establishes. While leveraging and brokerage refer to the characteristics of the process and of the structure, respectively, jointly they have a powerful ability to call for public attention and lead to change. As strategic tools, they allow for a renowned academician to attract the needed attention and gather the needed resources to lead change by his or her ability to connect the academic world with the public or social needs. Overall, leveraging and brokerage allow an ACL-motivated academician to become a unique motivator and energizer of social attention and social change.

Reliant on these concepts-cum-strategies, ACL defines not only the actors for this renewed university - namely, academicians and universities, personas, and institutions - but also requires that their drive to impact the public good rests on both commitment and leadership - that is, on both will and action. Commitment refers to a dedication to the cause of having science and academia deliver a social import. Because, as elaborated on in Chapter 3, ACL is defined and strategized in reference to corporate social responsibility (CSR), the choice of the term "commitment" is made intentionally to distinguish our proposed ACL model from the term "responsibility". In the context of universities, the term "responsibility" not only connotes accountability, moral capacity and reliability but is also tied to university programmes - value-added innovations, commercialization, teaching service, and developmental impact - which 
we later define as capturing the third (rather than ACL and thus fourth) academic mission (see Geschwind et al., 2019, p. 4; Zhang et al., 2018). Therefore, for our discussions, commitment stands for a sense of obligation where academic activities should deliver social impact, alongside achieving academic and scientific excellence.

Leadership, in our use here, means taking the initiative to set an agenda, organize a project, and recruit and guide others to partake in it. Importantly, both are weaved together to make ACL inherent to the university and to academic being. To what causes should academia commit and in which direction should it lead? On that, we are agnostic. Following Derek Bok's (Bok, 1982) discussion on how a university might prioritize among social challenges that require its input or intervention, we too think that the university's core commitment is to the development of knowledge and that all other responsibilities and commitments are subservient to it. Still, as we elaborate on throughout the book and especially in Chapter 4, ACL has the power to infuse the established academic missions - of teaching, research, and commercialization - with new themes and practices. Like the addition of the second academic mission of research to the first mission of infused teaching with research-driven themes, and much like the introduction of the third academic mission of production and technology transfer that redirected the missions of teaching and research in new (and sometime contested) directions, we think that the adoption of ACL as a fourth academic mission, by both academicians and universities, will open new venues for universitysociety exchange and will best engage academia with contemporary social challenges.

Discussion of ACL is embedded in a longer tradition of analysing and commenting on the public role of universities. This rich scholarly field uses many different terms to describe similar ideas or concepts - of which we chose particular ones to serve as the more accurate descriptors of the meanings we see. First, which organizations do we refer to? What is the field or sector we refer to? For the sake of consistency and clarity, throughout this book, we rely on the term "universities". Indeed, this term is commonly paired with "mission" when considering the strategic orientation, planning, and public role of higher education and academia. Nevertheless, while discussions of missions specifically refer to universities, the dramatic expansion of higher education, which also means the dramatic growth in the number and types of organizations that are accredited to award academic degrees, challenges this labelling. Indeed, the field of higher education is currently populated by colleges, teacher 
colleges, and technical colleges and universities; these various categories have become regulated by whomever the national accreditation body is; and such categorization formally recognizes only universities as accredited to award doctoral degrees, from which the accreditation process proceeds to specification based on the accreditation of curricular programmes. The result is an "alphabet soup" of sorts - with academic accreditation that is so specific by degree and discipline or field that most academicians, let alone lay people, cannot identify: D.Phil. or PhD; and MA and MSc, with a bewildering array of additional disciplinary academic accreditations in MBA, MFA, MSW, MLIS, or MDiv. ${ }^{1}$ There is one remedy to the confusion that results from the expansion and variation, and the accurate and most inclusive label for such variety is higher education organizations. However, the label of higher education organizations, or higher education institutions, orients the description towards education, sequencing with primary and secondary education and emphasizing teaching, learning, and skilling, whereas our intention is to comment on those organizations that are also committed to research and commercialization. Therefore, we employ the term "universities" to link our work with the scholarship on academic missions and to accentuate the plurality of academic missions in addition to teaching.

A second terminological choice that helps us maintain a conceptual focus throughout the book is specific to the naming of the academic missions. Alongside the well-encoded terms of "teaching" and "research" used to describe the first and second academic missions, discussions of the third academic mission commonly go under the title "entrepreneurial university". Enveloped in the term "entrepreneurial" are specific meanings that link the university to industry and to the production of knowledge that could be utilized for production and commercialization, and thus to technology transfer. However, these associations are not inherent to the notion of entrepreneurship; rather, entrepreneurship points more directly to the initiative and pursuit of social agents. Still, one of the claims that we advance in this book with regards to ACL is that initiative is central to the fourth mission; likewise, we argue that also inherent to ACL is that academic impact should reach outside the bounds of the university's gates. Therefore, to better articulate the defining features of the third mission and to differentiate it from ACL, which we advocate to be the fourth mission of universities, we herein refer to the third mission that is focused on commercialization. We find that this term best captures the third mission's orientation of the linear model of science-technology- 
industrial use, and production. It also best captures the utilitarian connotation of academic knowledge.

Third, we wrestled with the term that describes the object, or target, of ACL: throughout this book, we claim that, like the third mission, ACL aims to have universities shatter their image as an insular "ivory tower" and engage with, and impact, other societal institutions. However, several terms are used to describe the non-commercial engagement of universities with societal institutions outside their bounds: universities' social role, universities' public role, or universities' drive to contribute to the public good. After long discussions, we settled on the phrase "public role" of universities, and we use it throughout the book to capture its address of civic and communal matters, and yet it allows us to distinguish ACL from the ambiguity of the term "social" that is inherent to the now-common practice of corporate social responsibility (CSR), which we describe as a referent category for ACL.

These terminological choices are a primer to our discussion of and claims regarding ACL. They allow us to engage with the abundant scholarship on university-society relations over the course of a millennium, while also setting an agenda for academic engagement with contemporary and future societal challenges. We write this book with both analytic and advocacy intentions: by analysing the history of the societal missions of universities and seeking the sources for their inspiration, we define ACL as an opportunity and a programmatic tool for universities worldwide to refine their goals in terms of the public good, declare their agency in conquering social challenges, and institutionalize this new model of university-society relations through new practices, structures, and behaviours that embody this newly defined fourth mission of the university.

\section{THEMES AND STRUCTURE OF THE BOOK}

In this book, we ask the questions offered above in a way that is manifested in a combination of three main segments: evidence and examples, conceptual and analytical framing, and ideological advocacy and promotion. To provide evidence for emerging trends in the academic world, we collected a large sample of illustrative cases of what we term ACL (academic commitment and leadership), and we show evidence of some national and global processes that are associated with ACL. Towards the end of the book, we zoom into a specific site of ACL activities at the Hebrew University of Jerusalem, where the Hoffman Leadership and 
Responsibility Programme was established and developed with the aim of creating a community of practice to generate a greenhouse for ACL.

Throughout the book, we acknowledge the complexity and innovative nature of ACL that leads to areas of ambiguity and challenges of conceptual boundaries. While we understand that this is usually the nature of an emerging social paradigm, we aim to provide conceptual clarification and comparison of the framing of ACL to related paradigms. Our comparisons with other paradigms/missions go in two directions: an intra-institutional evolution (universities' historical perspective) and an inter-institutional evolution (comparison with CSR and influence of other societal dynamics). From an organizational and historical intra-institutional perspective, we provide an overview of the three established missions of universities - teaching, research, and production - and show how the emerging fourth academic mission of ACL compares with the long history of universitysociety relations. From an inter-institutional perspective, we compare between the CSR perspective in the for-profit organizational field to ACL in the academic field.

To design this ACL model and describe its principal features, we seek to engage with historical accounts of the university, discussions of publicness in organization studies, and studies of institutional dynamics of the organizational field of higher education. Overall, we draw on several fields of scholarships, on many tales of ACL-like initiatives and programmes from universities worldwide, and on the 14-year experience of the first author in leading an ACL doctoral programme at the Hebrew University of Jerusalem, namely the Hoffman Fellowship Programme.

Following this Introduction, Chapter 2 describes the historic roots of academia's public role. In this chapter, we review the frequently cited historiography of universities, which describes this institution's millennium of persistence as a sequence of responses to changing social conditions by adding academic missions. We then situate this historiography in a polemic essay about publicness, discussing how the general debate of university-society relations transmuted into a contemporary scheme for the higher education sector.

Chapter 3 seeks out sources for this more recent demand that universities express social responsibility. We argue that contemporary discussions of universities' social import and impact are translations of CSR models into academia. As universities are redefined as organizations - that is, formal, complex, and managed entities - and thus borrow governance models from other sectors, academia is also using CSR as a form of reference for its relations with society. We propose that ACL, 
while driven by CSR legitimacy, offers a model that is relevant to the longstanding, even ever-changing, public role of universities.

Chapter 4 offers an analytic description of ACL practices, describing types of ACL and examples for them. Our examples combine tales about universities and academicians: academic leaders - Einstein as an illustrative example - who are renowned scientists using their academic reputation to establish ACL attitudes, which later institutionalized as iconic models, and academic organizations, which institutionalized programmes and procedures that deliver ACL principles.

Chapter 5 outlines how the ACL model is and can be implemented for a university, arguing that ACL requires the constitution of an ACL-inspired academic community of practice. After describing the main features of a community of practice, we exemplify how team-building and establishing the normative culture became integrators that enhanced ACL practices. Due to the personal involvement of Oliver-Lumerman, the first author, in the founding and designing of a specific programme for ACL, Chapter 5 describes the Hoffman Leadership and Responsibility Programme that was established at the Hebrew University. It is a fellowship programme for excellent doctoral students from all disciplines that was dedicated to ACL through the facilitation of a community of practice. The characteristics of the programme, as well as the group processes that were facilitated, are described in Chapter 5.

Together, Chapters 5 and 6 offer confirmation for ACL practices: both on the organizational level, including organizational processes, and also on the individual level of academicians, we provide more in-depth illustration. Following Chapter 5, Chapter 6 celebrates a series of ACL-inspired projects at the Hoffman programme. With that, we provide inspiring examples of programmes, initiatives, and targets in the hope that these will serve as inspiration for future ACL initiatives. Chapter 6 provides examples of ten ACL projects that were developed by doctoral fellows of the Hoffman programme. These projects show the wide spectrum of cases where academic knowledge and understanding can be translated to societal needs in various directions.

Chapter 7 summarizes our arguments regarding ACL as the fourth mission of the university, also immersing them in discussions of the character and history of academia - central among them are the issues of autonomy and "academic freedom". In this chapter, we also advocate for ACL: we confess that aside from our analytic interest in institutional changes to universities, we also believe that ACL is an important mission for universities. Here, we let our "bias" show in the hope of directing our 
home institution and colleagues away from the prediction of the managerialist doom of academia, anticipating that the "real" fourth mission of universities is promotional, and towards accepting a fourth academic mission that harnesses the university for the expansion of the public good.

Overall, our book seeks to combine a description of the normative-cum-programme that is already budding in today's universities with the advocacy of ACL as a strategic and actionable model for 21 st-century universities. Today, yet again, questions about the future of universities are raised: is the university still a relevant social institution and, if so, how? Are academicians disconnected from "real life" and thus becoming irrelevant? Also, can universities keep up with the pace of social change and the magnitude of global society's grand challenges? We respond to such questions by offering our interpretation of which direction academia is moving these days and where it should proceed and expand to better articulate university-society relations in vision and in action.

\section{NOTE}

1. For the sake of not overshadowing the claim regarding the proliferation of academic degrees by these undecipherable acronyms, we add here a dictionary of sorts: Master of Business Administration (MBA), Master of Fine Arts (MFA), Master of Social Work (MSW), Master of Library and Information Studies (MLIS), and Master of Divinity (MDiv). 